\title{
Atomistic modeling study of a strain-free stress driven grain boundary migration mechanism
}

\author{
Liang Wan ${ }^{1,2,{ }^{*}}$, Akio Ishii ${ }^{1}$, Jun-Ping $\mathrm{Du}^{1,3}$, Wei-Zhong $\mathrm{Han}^{2}$, Qingsong $\mathrm{Mei}^{4}$, Shigenobu \\ Ogata $^{1,3, *}$ \\ ${ }^{1}$ Department of Mechanical Science and Bioengineering, Osaka University, Osaka 560-8531, Japan. \\ ${ }^{2}$ Center for Advancing Materials Performance from the Nanoscale, State Key Laboratory for \\ Mechanical Behavior of Materials, Xi'an Jiaotong University, Xi'an 710049, China. \\ ${ }^{3}$ Center for Elements Strategy Initiative for Structural Materials, Kyoto University, Kyoto 606-8501, \\ Japan. \\ ${ }^{4}$ School of Power and Mechanical Engineering, Wuhan University, Wuhan 430072, China
}

*Corresponding authors. Email addresses: liangwan5@gmail.com; ogata@me.es.osaka-u.ac.jp

A recent experiment (Scripta Mater., 65:990, 2011) shows that the $\Sigma 7\{132\} /\{132\}$ grain boundary in Al can migrate under external stress but produces no strain. Here, based on a bi-crystallographic analysis, an atomic shuffling path was identified as the feasible mechanism for this grain boundary migration. By a density functional theory calculation, it reveals that the enthalpy barrier of this atomic shuffling path increases by external shear stress applied with shear of the grain boundary along the tilt axis $\langle 111\rangle$, which is in good agreement with experimentally measured shear-direction-dependence of activation enthalpy for this grain boundary migration.

Keywords: Grain boundary migration; Density functional theory (DFT); Coincidence lattice; Grain boundary structure; Atomic shuffling motion 
For polycrystalline materials, grain boundary $(G B)$ migration is the major structural transformation process that contributes to the grain growth phenomena [1, 2]. Traditionally, GB migration is thought to occur only at medium or higher temperatures $\left(>0.4 T_{m}, T_{m}\right.$ is the melting temperature) by thermally assisted atomic shuffling motions in the GB zone [2]. Some efforts have been made to characterize the patterns of the motions of atoms involved, e.g., there are suggestions that a 'stringlike' motion of atoms [3] or rotation of cluster of atoms [4] in GB zone can be identified for migration of some particular GBs. It appears that the specific pattern of the atomic shuffling motions on migration of GB is a case-by-case basis because atomistic structure of the GBs are different one another, and different GBs can therefore display quite different temperature dependence of mobility [5]. Nevertheless, for a 'random' GB, this kind of atomic shuffling motions is generally random in nature as well: motions of atoms in the GB zone are less coordinated, and migration of the GB is thermally assisted without any transformational strain produced. However, in the past decade, it has been widely reported that, for small grained polycrystalline materials, GB migration can be induced by external stress at room temperature or even in cryogenic loading conditions [6-11]. Further investigations indicate that GB migration in these circumstances can be well accounted for by the shear coupled GB motions wherein the bi-crystallographic lattice structure of GB plays an important role [12]. These findings signify that, the stress driven GB migration behavior can be a typical characteristic of GBs, especially for GBs in small grained polycrystalline materials [12].

Compared with the thermally assisted migration of GB by random atomic shuffling motions 
in the GB zone, the shear coupled GB migration features a shear strain produced in the volume swept by the migration motion of the $\operatorname{GB}[12,13]$. It is worth to mention that, a recent study shows that the basal/prismatic (B/P) boundary in Mg can migrate under uniaxial stress normal to the boundary [14]. Migration of the $B / P$ boundary in this case is accomplished via a 'unit-cell-reconstruction' mechanism [15], and it produces a tetragonal strain by the migration of the boundary [14]. For both the shear coupled GB migration and the migration of the $\mathrm{B} / \mathrm{P}$ boundary in $\mathrm{Mg}$, atomic shuffling motions in the $\mathrm{GB}$ zone can be identified as a complementary process on migration of the $G B[14,16,17]$. In short, the stress driven GB migration is generally characterized by a transformational strain produced in the volume swept by the motion of GB together with complementary atomic shuffling motions in the volume. It is therefore quite similar to the martensitic phase transformation processes in materials $[18,19]$.

However, an Al bicrystal experiment carried out recently by Molodov et al. [20] seems to provide an exceptional case. In their experiment, the $\Sigma 7\{132\} /\{132\}$ symmetric tilt coincidence site lattice (CSL) boundary (hereafter termed as $\Sigma 7\{132\}$ GB for short) in Al was shown to migrate under external stress at temperatures of $600 \sim 700 \mathrm{~K}$. For external stress applied with shear of the GB along three different directions in the GB plane, no strain was measured in the GB migration processes, and the activation enthalpy measured for the GB migration shows a strong shear-direction-dependent characteristic. Although it has been proposed that a dislocation model of GB [13] can be used to interpret this GB migration phenomena [20], and also a two-step atomic shuffling process has been suggested [21], the 
following questions remain: (i) how the GB migration occurs on the atomistic level; (ii) why the GB migrates under external stress but produces no strain; (iii) why the activation enthalpy for the GB migration is shear direction dependent. It is the purpose of the present work to provide an atomistic level understanding of this mysterious GB migration phenomena and to answer these questions.

For the $\Sigma 7\{132\} \mathrm{GB}$ in Al, its bi-crystallographic structure can be analyzed first. Figure 1a shows the CSL structure of this GB, with both the 'real sites' (spheres) of neighboring grains and the extrapolated 'virtual sites' (dashed circles) of the two grains displayed. It can be seen that the primitive cell of the CSL of this GB has exactly seven atoms, which conforms to the bi-crystallographic theory of GBs [1, 22]. By selecting the boundary plane in the position as indicated by the black dashed line in Figure 1a, the structure of the $\Sigma 7\{132\}$ GB obtained can be illustrated by the blue lines sketched. To check whether the bi-crystallographic theory can describe the atomistic structure of this type of GB in Al or not, atomistic modeling of the structure of the GB in $A l$ with an embedded atom method (EAM) potential [23] was performed by using LAMMPS [24]. A bicrystal model with the same lattice orientations of neighboring grains as in Figure 1a was constructed. Periodic boundary condition (PBC) was applied to the dimensions parallel to the GB plane, while free surface boundary condition was used on the dimension perpendicular to the GB plane. A procedure the same as that of the references $[16,17]$ was adopted for exploration of the minimum energy structure of the GB. 
Figures $1 \mathrm{~b}$ and $1 \mathrm{c}$ show the simulated atomistic structure of the $G B$ in $A l$ by the EAM potential. As can be demonstrated by the blue lines in Figure $1 \mathrm{~b}$, the overall atomistic structure of the GB simulated agrees pretty well with the bi-crystallographic theory description as in Figure 1a. On the other hand, the dashed ovals marked in Figure $1 \mathrm{~b}$ indicate that, the atoms in the layer closest to the boundary plane have a position biased from that of the bi-crystallographic lattice sites shown in Figure 1a. Figure 1c shows that, the neighboring grains of the GB in $A$ l have a small relative displacement along the tilt axis $<111>$ as compared to the CSL structure. Nevertheless, as a good approximation, the atomistic structure of the GB in Al can be viewed as a result of displacement of the 'real sites' in layer just below the boundary plane in the CSL according to the vectors indicated by the bold arrows in Figure 1a.

Based on the above analysis, the bi-crystallographic lattice structure of the $\Sigma 7\{132\}$ GB can be slightly modified. Figure 1d shows the bi-crystallographic lattice as in Figure 1a, but with the 'real sites' in lattice plane 0 (now the grey shaded circles) interchanged with the 'virtual sites' on this plane (now the spheres marked by $\times$ ) according to the bold arrows in Figure 1a. By a careful examination of the modified lattice, an atomic shuffling path as demonstrated by the arrows in Figure 1d can be figured out. It shows that, with the atoms on the 'real sites' move to the 'virtual sites' following the vectors indicated in the lattice, the GB (see the blue solid lines) can migrate from the lattice plane 1 up to the lattice plane 8 (see the blue dashed lines). One can find that the atoms involved in this shuffling path (i.e., atoms between the lattice plane 0 and lattice plane 8) move only a small distance around in a somewhat 
coordinated way, and they do not jump between the neighboring $\{111\}$ planes along the tilt axis $<111>$. It is therefore very likely that this atomic shuffling path can give a comparatively low energy barrier. Importantly, by this single migration step, there is no relative displacement of the atoms above the lattice plane 7 to those below the lattice plane 0 , and the atomistic structure of the GB fully recovers after this migration step. It implies that migration of the GB following this shuffling path does not produce strain in the end. This makes it a rather good candidate of the possible atomistic mechanism that accounts for the GB migration motion observed in the Al bicrystal experiment mentioned above.

To check this assumption, a first-principles calculation of the minimum energy path (MEP) of the above-identified atomic shuffling path for migration of the $\Sigma 7\{132\}$ GB in Al was performed with the density functional theory (DFT) method. The DFT calculation was performed using VASP [25] with a Perdew-Wang generalized gradient approximation functional [26] and projector-augmented wave potentials [27] for Al $\left(3 s^{2} 3 p^{1}\right)$. An energy cutoff of $240 \mathrm{eV}$ was used for the plane wave expansion. The energy convergence criteria of the electronic and ionic structure relaxations were set to $10^{-8}$ and $10^{-3} \mathrm{eV}$, respectively. Figure 2a shows the bicrystal model used in the DFT calculation. The simulated structure of the GB obtained by atomistic modeling with EAM potential was used as the input configuration. Three dimensional PBC was adopted in the bicrystal model. In dimensions parallel to the GB plane, a single period of the primitive cell of CSL was used. For the dimension perpendicular to the GB plane, the length of the model was made fairly large to avoid interference of the second boundary introduced by the PBC. The total number of atoms is 50 in the bicrystal 
model. A Monkhorst-Pack k-point mesh [28] of $8 \times 8 \times 3$ was used. The MEP calculation was performed using the drag method [29], with a constant external stress applied on the model for each image on the path [30]. In the drag method, a scalar shuffling degree (SSD) was defined to characterize the overall atomic shuffling distance along the shuffling path [31]. Fourteen images along the atomic shuffling path were used.

Figure 2a shows that, the optimized structure of the GB in Al obtained by the DFT method is almost the same with that by using the EAM interatomic potential (see Fig. 1b). Figure 2b shows variation of the potential energy of the model for the images along the MEP calculated by the DFT method. Here, the external stress of the model was set to zero for each image along the shuffling path. The zero external stress means that the size and shape of the model can freely relax. The shear strain of the model produced along the atomic shuffling path can also be calculated by measuring the shape change of the model. Variation of two shear strain components of the model, with shear of the GB along [111] (the tilt axis) and $[\overline{5} \overline{14}]$ (perpendicular to the tilt axis on GB plane), is shown in Figure $2 \mathrm{~b}$ as well (note that all the lattice directions in this article are given with the upper grain as the reference lattice). The atomistic structure of the GB for all images along the MEP can also be obtained. Figures $2 \mathrm{c}$ and $2 \mathrm{~d}$ show the atomistic structures of the GB for an intermediate state and the saddle state (both are marked by the arrows in Fig. 2b), respectively. A video which illustrates the structure evolution of the GB along the MEP is provided as supplementary online information. 
Several aspects of the MEP calculated need to be pointed out. First, the energy barrier calculated for the atomic shuffling path is around $0.09 \mathrm{eV}$. This is a rather low value, as compared with the experimentally measured activation enthalpy of $0.67 \sim 1.3 \mathrm{eV}$ for the GB migration [20]. However, consider that there is only one period of the primitive cell of CSL in the dimensions parallel to the GB plane in the DFT calculation (see Fig. 2a), it is very likely that some kind of loop-of-GB-step nucleation and expansion process following this atomic shuffling path is functioning for GB migration in experiment, similar to the case for which the nucleation and expansion of GB DSC dislocation loop result in shear coupled GB migration motion $[1,16,17,32]$. The critical length of the migrated step line can be perhaps a few tens periods of the primitive cell of CSL in the experimental condition, for which the enthalpy barrier can then be plausibly compared with the activation enthalpy of GB migration measured experimentally. However, a detailed thermodynamic analysis and precise calculation of this loop-of-GB-step nucleation and expansion process is beyond the scope of this article. Nevertheless, the fairly low energy barrier indicates that the atomic shuffling motion as illustrated in Figure $1 \mathrm{~d}$ can be readily activated for this type of GB in Al. Second, as can be seen in the potential energy curve in Figure $2 b$, during the migration of the GB following this shuffling path, it first transformed to an intermediate state. One can see in Figure $2 c$ that the intermediate state corresponds to a GB structure which is mirror symmetrical to that of the state before migration (see Fig. $2 a$ ). Figure $2 b$ shows that this intermediate structure has almost the same potential energy with that of the state before migration. Third, Figure $2 \mathrm{~b}$ indicates that, migration of the GB following this shuffling path does not produce any transformational strain in the end, which conforms to the 
bi-crystallographic analysis of the shuffling path in Figure $1 \mathrm{~d}$ as mentioned above. However, one can find in the shear strain curves in Figure $2 b$ that, there is finite shear strain of the model with shear of the GB along the tilt axis [1111] produced in the intermediacy of the MEP. This can be clearly demonstrated by the supplementary video which shows that the model shears forth and back along the $[\overline{1} 11]$ during migration of the GB.

Migration of the GB following this atomic shuffling path does not produce strain in the end of the shuffling path, and yet results in finite shear strain with shear of the GB along the tilt axis [111] in the intermediacy of the shuffling path, is interesting and can be further examined. With shear strain produced in the intermediacy of the path, work can be done in the model by external shear stress applied with shear of the GB along the [111] correspondingly. This can normally result in a change of the enthalpy of the model. Indeed, a further DFT calculation of the MEP with finite external shear stress applied on the model for shear of the GB along [111] reveals a shear-stress-dependent enthalpy profile of the MEP as shown in Figure 3a. By comparison, for shear stress applied with shear of the GB along $[\overline{5} \overline{14}]$, which is perpendicular to $[\overline{1} 11]$ on the GB plane, Figure $3 \mathrm{~b}$ indicates that no such stress dependent behavior can be recognized. This agrees with the red curve in Figure $2 b$ that, there is almost no shear strain component produced with shear of the GB along $[\overline{5} \overline{1} \overline{4}]$ on the MEP when zero external stress is applied on the model. It should be mentioned that, for external shear stress applied with shear of the GB along $[1 \overline{1} \overline{1}]$, the minimum energy structure of the GB can be changed by a certain level of stress applied. Analysis shows that the new minimum energy structure is right the mirror symmetrical configuration of the 
original GB as shown in Figure 2c.

From Figures $3 a$ and $3 b$, the enthalpy barrier for this atomic shuffling path with different external shear stresses applied on the model can be evaluated. Figure $3 c$ shows the variation of the enthalpy barrier with different level of shear stresses applied as calculated by DFT. It reveals that the enthalpy barrier for this atomic shuffling path increases by shear stress applied with shear of the GB along [1]11], while it almost does not change by shear stress applied with shear of the GB along $[\overline{5} \overline{1} \overline{4}]$. Figure $3 \mathrm{~d}$ illustrates the three shear directions adopted in the experiment performed by Molodov et al. [20], with their experimentally measured activation enthalpy indicated as well. One can notice that, the closer the shear direction to the $[\overline{1} 11]$, the higher the measured activation enthalpy. From the Figures $3 \mathrm{c}$ and $3 d$, it can be seen that there is a good agreement between the DFT calculated stress dependent change of the enthalpy barrier for this atomic shuffling path and the experimentally measured shear-direction-dependence of activation enthalpy for GB migration. This agreement lends us further credence that the GB migration as observed in experiment by Molodov et al. [20] can be well explained by the atomic shuffling mechanism as indicated in Figure $1 \mathrm{~d}$. As analyzed above, the experimentally measured shear-direction-dependence of activation enthalpy for GB migration should be attributed to the fact that finite shear strain with shear of the GB along the tilt axis $<111>$ is produced in the intermediacy but not at the end of the atomic shuffling path.

For the experimentally observed migration of the $\Sigma 7\{132\} \mathrm{GB}$ in $\mathrm{Al}$ [20], the thermodynamic 
driving force for GB migration still needs to be figured out. Since there is no strain produced ultimately by migration of the GB, no net work can be done by the external stress applied to provide the driving force for the GB migration. Meanwhile, Al has a small elastic anisotropy factor in a wide temperature range [33, 34]. Given that the bicrystal experiment as carried out by Molodov et al. [20] was performed with an external stress less than $1 \mathrm{MPa}$, it can be calculated that the elastic strain energy difference of a 7-atom cell of Al with the two orientations of neighboring grains is less than $10^{-6} \mathrm{eV}$. We reckon that it is also unlikely that the elastic strain energy difference of the neighboring grains can drive the migration of the GB. Further investigation is therefore required for the confirmation of thermodynamic driving force for the migration of the GB as shown in the experiment [20].

In summary, the atomistic mechanism for the strain-free stress driven GB migration of the $\Sigma 7$ $\{132\}$ GB in $A$ l as observed in a previous experiment has been examined in depth. By an analysis of the bi-crystallographic lattice structure of this GB, an atomic shuffling path was identified to be the feasible mechanism for this strange GB migration behavior. MEP calculation of this atomic shuffling path by DFT method shows that, due to the finite shear strain with shear of the GB along the tilt axis $<111>$ produced in the intermediacy but not at the end of the atomic shuffling path, the enthalpy barrier of the path increases by external shear stress applied with shear of the GB along the tilt axis $\langle 111\rangle$, which is in good agreement with the experimentally measured shear-direction-dependence of activation enthalpy for the GB migration. We conclude that the experimentally observed GB migration behavior can be well explained by the proposed atomic shuffling mechanism. The strain-free 
stress driven GB migration of the $\Sigma 7\{132\} \mathrm{GB}$ in $\mathrm{Al}$ provides another example that stress driven GB migration is directed by the bi-crystallographic lattice structure in the GB.

Financial support from National Science Foundation of China (Grant Nos. 51201127, 51371128) is gratefully acknowledged. S.O. acknowledges support from the Elements Strategy Initiative for Structural Materials (ESISM). W.Z.H. would like to thank the support of Youth Thousand Talents Program of China.

\section{References}

[1] A.P. Sutton, R.W. Balluffi, Interfaces in Crystalline Materials, Clarendon Press, Oxford, 1995.

[2] G. Gottstein, L.S. Shvindlerman, Grain boundary migration in metals: thermodynamics, kinetics, applications, CRC press2009.

[3] H. Zhang, D. Srolovitz, J. Douglas, J. Warren, Physical Review B 74(11) (2006).

[4] C. O’Brien, S. Foiles, Journal of Materials Science 51(14) (2016) 6607-6623.

[5] E.R. Homer, E.A. Holm, S.M. Foiles, D.L. Olmsted, Jom-Us 66(1) (2014) 114-120.

[6] K. Zhang, J.R. Weertman, J.A. Eastman, Applied Physics Letters 85(22) (2004) 5197.

[7] D.S. Gianola, S. Van Petegem, M. Legros, S. Brandstetter, H. Van Swygenhoven, K.J. Hemker, Acta Materialia 54(8) (2006) 2253-2263.

[8] M. Legros, D.S. Gianola, K.J. Hemker, Acta Materialia 56(14) (2008) 3380-3393.

[9] T.J. Rupert, D.S. Gianola, Y. Gan, K.J. Hemker, Science 326(5960) (2009) 1686-90.

[10] Y. Zhang, J.A. Sharon, G.L. Hu, K.T. Ramesh, K.J. Hemker, Scripta Materialia 68(6) (2013) 424-427.

[11] B. Wang, M.T. Alam, M.A. Haque, Scripta Materialia 71 (2014) 1-4. 
[12] L. Wan, W. Han, K. Chen, Scientific reports 5 (2015) 13441.

[13] J.W. Cahn, Y. Mishin, A. Suzuki, Acta Materialia 54(19) (2006) 4953-4975.

[14] B.-Y. Liu, J. Wang, B. Li, L. Lu, X.-Y. Zhang, Z.-W. Shan, J. Li, C.-L. Jia, J. Sun, E. Ma, Nature communications 5 (2014) 3297.

[15] B.-Y. Liu, L. Wan, J. Wang, E. Ma, Z.-W. Shan, Scripta Materialia 100 (2015) 86-89.

[16] L. Wan, S. Wang, Physical Review B 82(21) (2010).

[17] L. Wan, J. Li, Modelling and Simulation in Materials Science and Engineering 21(5) (2013) 055013.

[18] J.W. Christian, The theory of transformations in metals and alloys, Newnes2002.

[19] D.A. Porter, K.E. Easterling, M. Sherif, Phase Transformations in Metals and Alloys, (Revised Reprint), CRC press2009.

[20] D.A. Molodov, T. Gorkaya, G. Gottstein, Scripta Materialia 65(11) (2011) 990-993.

[21] F. Ulomek, V. Mohles, Acta Materialia 103 (2016) 424-432.

[22] H. Grimmer, W. Bollmann, Warringt.Dh, Acta Crystallogr A A 30(Mar) (1974) 197-207.

[23] Y. Mishin, D. Farkas, M.J. Mehl, D.A. Papaconstantopoulos, Physical Review B 59(5) (1999) 3393-3407.

[24] S. Plimpton, J Comput Phys 117(1) (1995) 1-19.

[25] G. Kresse, J. Furthmüller, Physical review B 54(16) (1996) 11169.

[26] J.P. Perdew, J. Chevary, S. Vosko, K.A. Jackson, M.R. Pederson, D. Singh, C. Fiolhais, Physical Review B 46(11) (1992) 6671.

[27] G. Kresse, D. Joubert, Physical Review B 59(3) (1999) 1758.

[28] H.J. Monkhorst, J.D. Pack, Physical review B 13(12) (1976) 5188.

[29] H. Jónsson, G. Mills, K.W. Jacobsen, CLassical and Quantum Dynamics in Condensed Phase 
Simulations, 1998, pp. 385-404.

[30] S. Ogata, J. Li, S. Yip, Physical Review B 71(22) (2005) 224102.

[31] A. Ishii, J. Li, S. Ogata, International Journal of Plasticity 82 (2016) 32-43.

[32] L. Wan, S. Wang, Modelling and Simulation in Materials Science and Engineering 17(4) (2009) 045008.

[33] D. Gerlich, E.S. Fisher, J Phys Chem Solids 30 (1969) 1197-1250.

[34] H.H. Pham, M.E. Williams, P. Mahaffey, M. Radovic, R. Arroyave, T. Cagin, Physical Review B 84(6) (2011) 


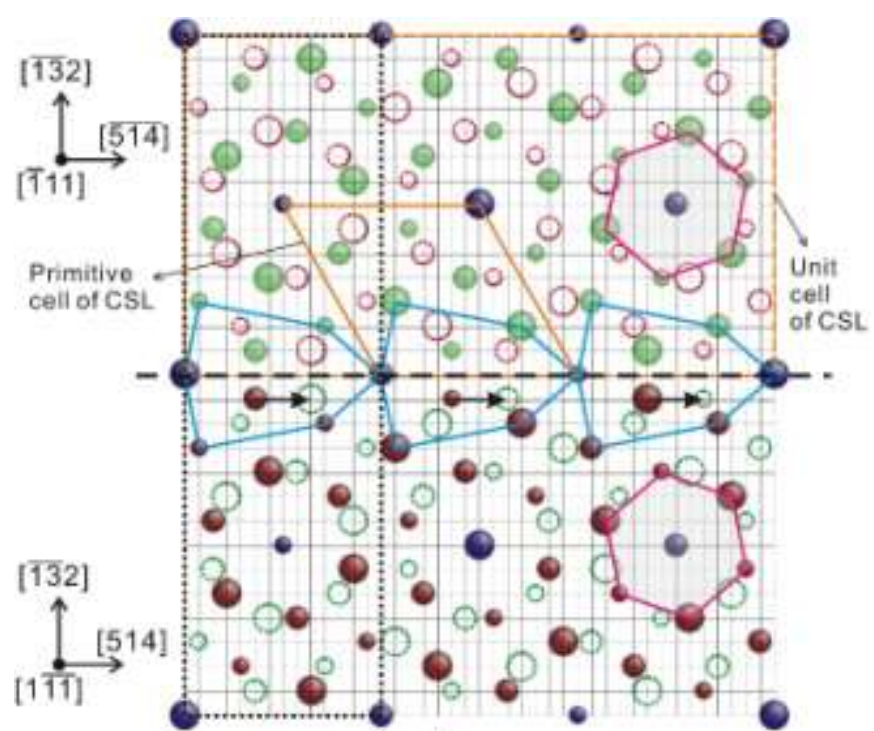

(a)

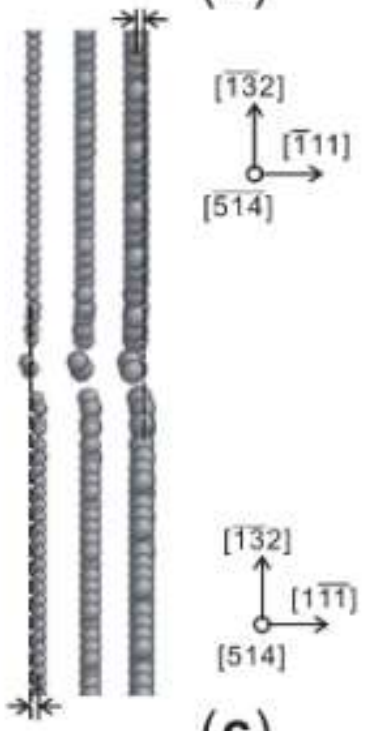

(c)

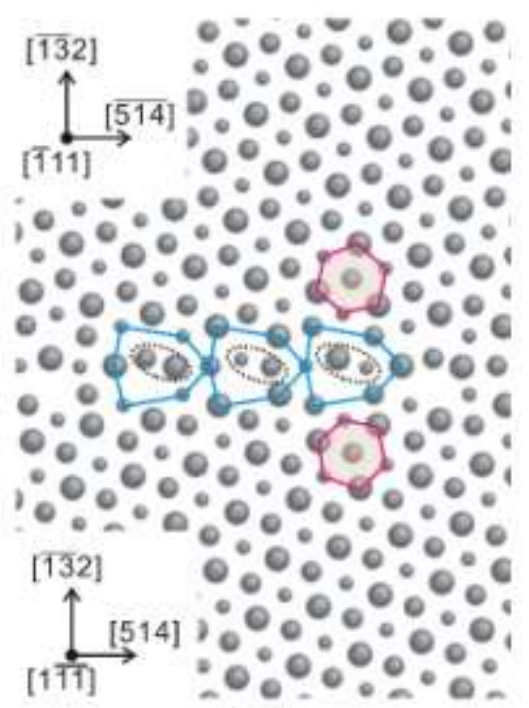

(b)

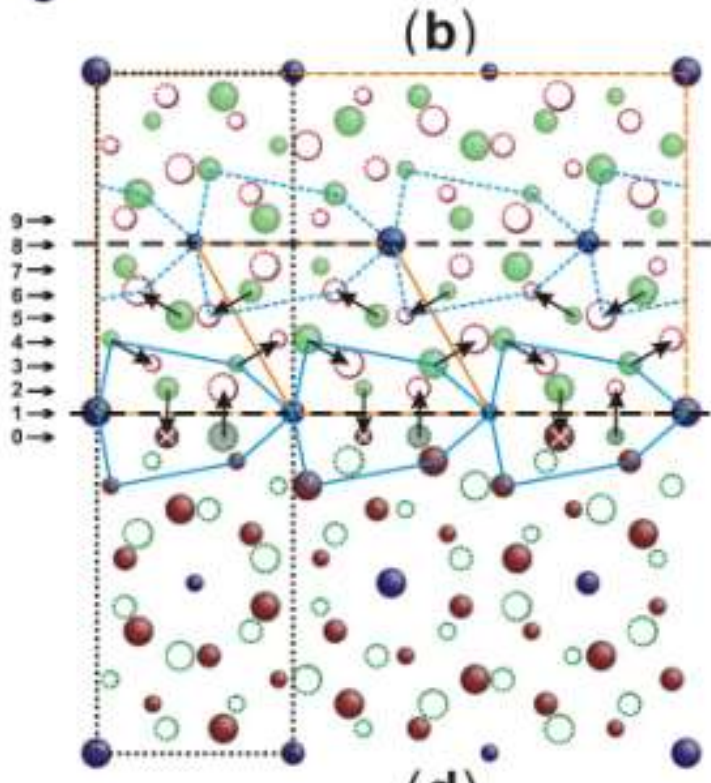

(d)

Figure 1. Bi-crystallographic analysis of the $\Sigma 7\{132\} \mathrm{GB}$ in Al and the simulated GB structure. (a) is the CSL of the $\Sigma 7\{132\}$ GB in FCC crystal viewed along the tilt axis $<111>$. Green and dark red spheres represent lattice sites of the upper and lower grains ('real sites'), respectively. Green and red dashed circles represent the extrapolating of the lattices of the upper and lower grains to the other side of the boundary plane ('virtual sites'), respectively. The GB plane is indicated by the black dashed line. The dark blue spheres are the coincident sites. The fine grid is the displacement shift complete (DSC) lattice. Spheres/circles of three sizes distinguish the three different $\{111\}$ lattice layers along the tilt axis $\langle 111\rangle$, with the larger ones closer to the reader. (b) and (c) show the simulated atomistic structure of this type of GB in Al by EAM potential viewed along the tilt axis $<111>$ and perpendicular to the tilt axis, respectively. The size of spheres is also used to distinguish the three different $\{111\}$ atom layers along the tilt axis $<111>$. (d) is the CSL of the GB as in (a), which shows the atomic shuffling path for the GB migration. In (d), ten adjacent lattice planes (marked as 0 9) parallel to the boundary plane are marked. 
(a)

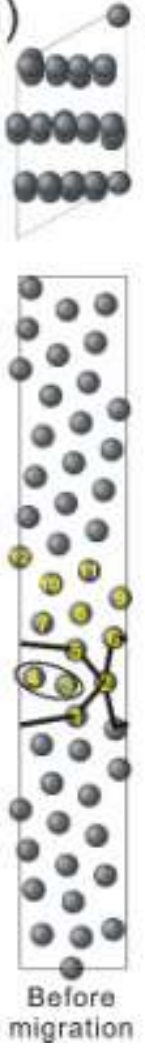

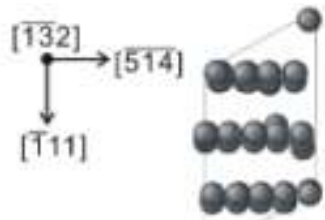

[132]

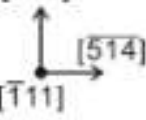

[132]

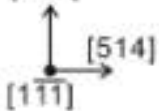

$\stackrel{[514]}{\longrightarrow}$

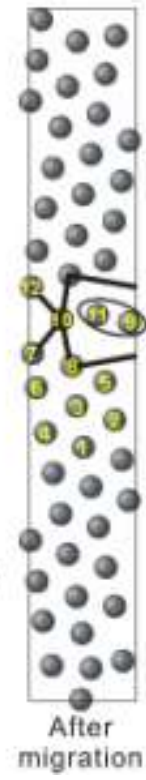

(b)

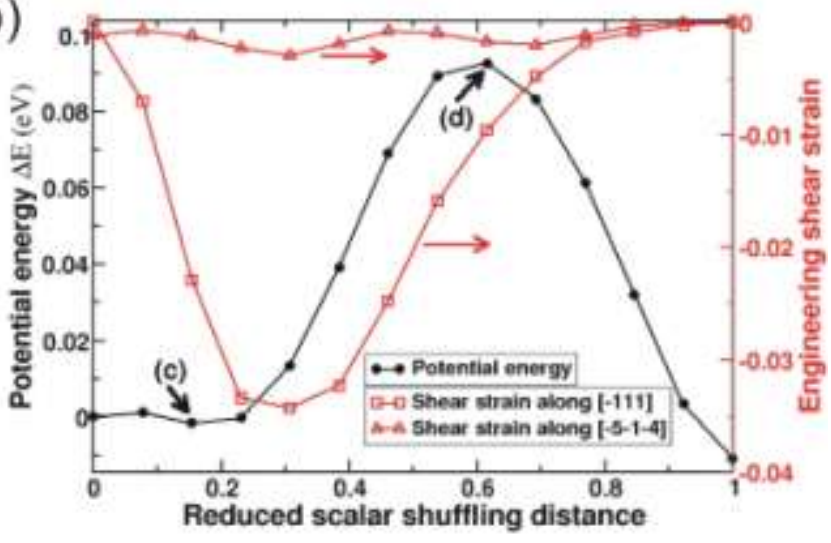

(c)

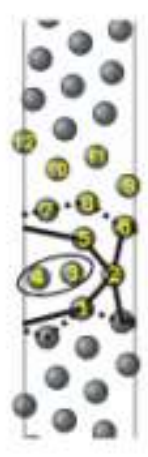

(d)

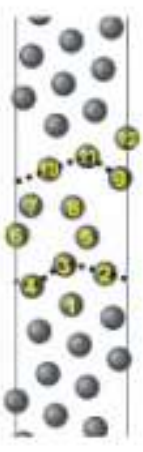

Figure 2. DFT calculation of the MEP of the atomic shuffling path for the GB migration in AI. (a) The bicrystal model used for the DFT calculation of the MEP. The left and right panels are the configurations of the bicrystal model before and after migration of the GB, respectively. For both configurations, views of the model together with the simulation cell along the $G B$ normal (upper panel) and tilt axis $<111>$ (lower panel) are illustrated. Both configurations were obtained by a geometry optimization using the DFT method. The GB is indicated by the bold solid lines sketched similar to that in Figure 1b. See the dotted rectangle in Figs $1 \mathrm{a}$ and $1 \mathrm{~d}$ for the periodicity of the model parallel to the GB plane. (b) Variation of the potential energy and engineering shear strain of the model for images along the MEP with zero external stress applied on the model. Two shear strain components with shear of the GB along [ $[\overline{1} 11]$ and $[\overline{5} \overline{1} \overline{4}]$ are shown. The abscissa axis is given by the reduced scalar shuffling distance of the images on the atomic shuffling path. (c) and (d) are views of the GB along the tilt axis $<111>$ for an intermediate state and the saddle state on the MEP as indicated by the corresponding arrows in (b), respectively. In (a), (c) and (d), 12 atoms are marked by numbers 1 12 to illustrate the movement of the atoms in the GB zone during the GB migration. Note that atoms will be wrapped back to the opposite side of the cell on crossing the boundary of the cell due to the application of PBC on the model. 


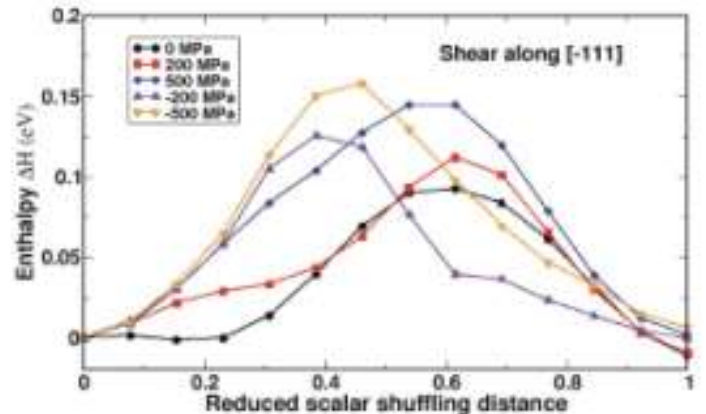

(a)

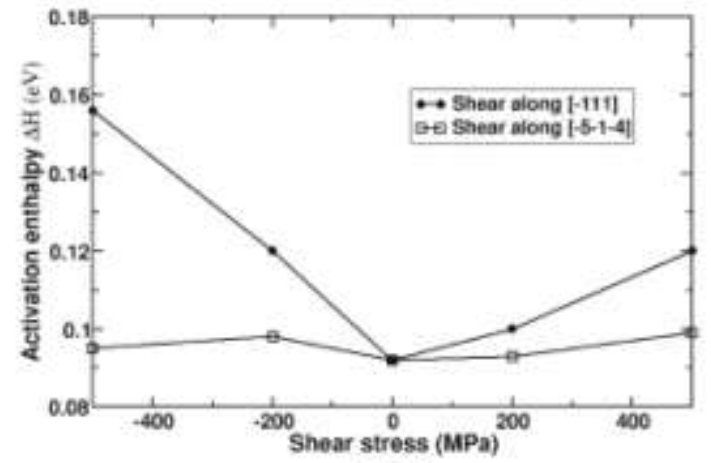

(C)

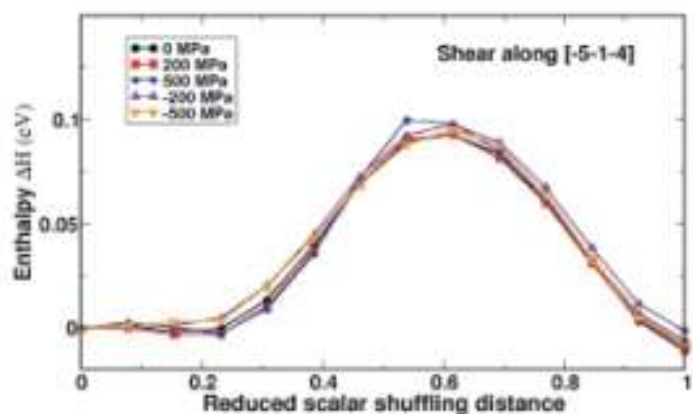

(b)

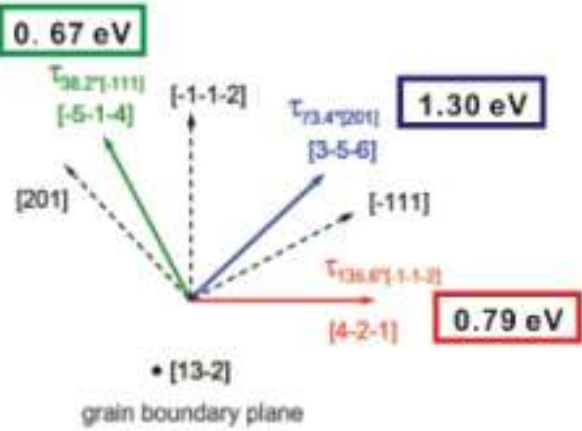

(d)

Figure 3. Comparison of stress dependence of the enthalpy barrier for the atomic shuffling path calculated by DFT method with the experimentally measured shear-direction-dependence of activation enthalpy for GB migration. (a) and (b) are variation of the enthalpy of the model in Fig. 2a along the MEP for different external shear stress levels applied on the model with shear of the GB along the $[\overline{1} 11]$ and $[\overline{5} \overline{1} \overline{4}]$, respectively. (c) is the enthalpy barrier of the atomic shuffling path at different shear stresses, evaluated from (a) and (b). (d) is the three shear directions adopted in the experiment performed by Molodov et al. [20], with their experimentally measured activation enthalpy for GB migration indicated aside correspondingly. 


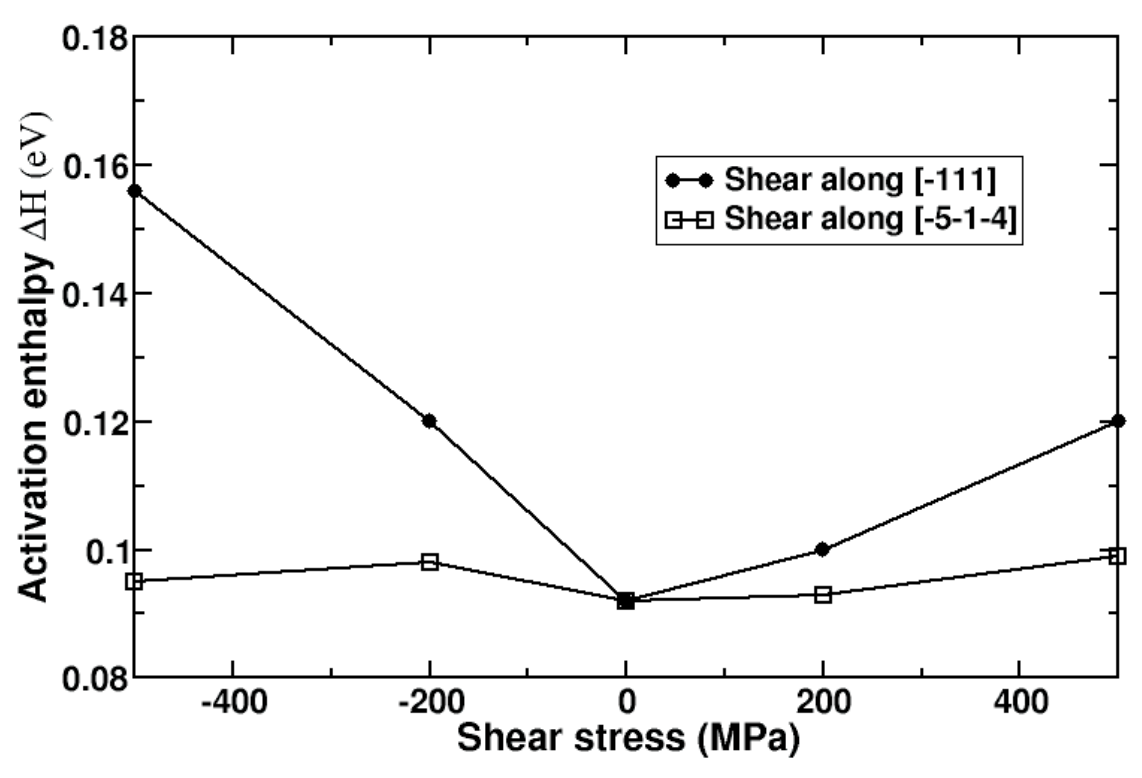

